\title{
Introduction to the Special Issue: Intersections of Artificial Intelligence and Community Well-Being
}

\author{
R. Phillips ${ }^{1}$ - L. Musikanski ${ }^{2}$ - M. Manson ${ }^{3}$ - J. Bradbury ${ }^{2}$ - L. Fraizer ${ }^{4}$. \\ B. Rakova ${ }^{5}$ - N. DePalma ${ }^{6}$ - A. Smart ${ }^{7}$
}

Accepted: 22 October 2020 / Published online: 19 November 2020

(C) Springer Nature Switzerland AG 2020

Welcome to the Intersections of Artificial Intelligence (AI) and Community WellBeing special issue for the International Journal of Community Well-Being. The intent of this special issue is to inform, inspire and aid researchers, academics, practitioners, policy makers and activists in understanding the scope of implications of AI for community well-being. Our underlying mission is to contribute to a present and future where AI contributes to the well-being of communities, humans, and the planet through community-based platforms. There are many challenges in constructing a special issue about an evolving subject and its prevalence as a field of research in academic circles or as a department, goal, or agency in business and government. The impact of AI on community well-being continues to be significant despite the dearth of studies and research on the subject that represents profound impacts on the lives of every human on the planet, our communities, and our ecosystems. We began this special issue before the advent of the pandemic. With its impact on our communities and lives along with growing reliance on technology including AI, we feel a particular need for research and investigation into the intersections of AI and community well-being. For these reasons, we accepted the challenge of forming this special issue.

The articles comprising this issue demonstrate the nascent state of collective understanding of the implications of AI on community well-being. They also demonstrate that there is much to learn about the impact of AI regarding engagement, involvement, and preservation of communities and their well-being. Our aims in publishing this special issue are many. Primarily, we hope that academics and practitioners in the fields of community well-being and AI, as well as other areas of research and practice, will use this special issue as a starting point in making connections between these two areas for developing more understanding about challenges and opportunities.

Chief Guest Special Issue Editor: Rakova, B.

Guest Special Editors: Manson, M., Musikanski, L., Phillips, R., Fraizer, L., Bradbury, J., De Palma, N., Smart, A.

R. Phillips

rphillips@purdue.edu

Extended author information available on the last page of the article 
Making these connections will require interdisciplinary, transdisciplinary and crosssector undertakings, given the scale and magnitude of current and potential impact across the planet. The lack of exploration across perspectives as represented in disciplinary studies or siloed approaches will not help move society forward given the inherently inter/trans/cross-disciplinary nature of the challenges at hand. Instead, a systems change approach will be needed to parse through multiple, complex and interconnected dimensions of influence. For example there are, in very general terms, four different entry points for eliciting change in a system: symptoms, patterns, design and values (Maani and Cavana 2007). The most common entry point to solve a problem is typically symptoms, yet the most powerful entry point may be values, as changing values can cause a large change to a system (Musikanski et al. 2020a). Values can certainly play a part in making connections between AI and community well-being, particularly since the latter can be assessed or measured with indicators, which are essentially reflective of the values of a population (OECD 2019; Phillips and Wong 2016). Design will also play a vital role - as AI is integrated more fully, it will be crucial to consider community well-being impacts; likewise, patterns help to understand if a system is healthy or not, and reveal values held by those in systems (Musikanski et al. 2020a, p. 23).

We also set the foundation for the special issue with an earlier work this year, "Artificial Intelligence and Community Well-being: A Proposal for an Emerging Area of Research" (Musikanski et al. 2020b). This article identifies three areas helpful in understanding and developing research at the intersection of AI and community wellbeing. These areas are: (1) the formation of a means of measuring and assessing the impacts of AI on communities, (2) the engagement of communities in the development, deployment and management of AI, and (3) the creation of AI to safeguard and improve community well-being from social, ecological, economic and other threats such as climate change, dismantling of democracy, destabilizing economic inequality, and decimation of natural resources (Musikanski et al. 2020b). These three areas represent many issues, challenges, and opportunities across disciplines and practice.

Before we present the synopses of the articles, let us discuss some parameters of community well-being which are likely less well-known than is artificial intelligence (especially given its use in smart phones with Siri and other applications). The Organization for Economic Co-operation and Development (OECD) defines wellbeing as: "people's living conditions and quality of life today (current well-being), as well as the resources that will help to sustain people's well-being over time (natural, economic, human and social capital)" (OECD 2018, p. 2). Others imply that well-being arises "in the context of society and social collectivity" (McGregor 2007, p. 318); and its evolution is via "set of interlocking issues and constraints...embedded in a dynamic social context" (Phillips and Wong 2017, p.1) and "embedded with multidimensional values including the economic, social, and environmental aspects that impact people" (Phillips and Wong 2017, p. xxix). In our articles setting the foundation for this special issue, we note that community well-being includes the following domains: (1) community, (2) culture, (3) economy-standard of living (which includes housing, food, transportation and information and communication technology), (4) education, (5) environment, (6) government, (7) health, (8) psychological well-being, (9) subjective well-being and affect, (10) time balance and (11) work (Musikanski et al. 2020a, p. 41). 
Just with this brief introduction, it is easy to see it is multifaceted and touches on many aspects of living together in societies of place and interest.

\section{Synopses of Articles}

The articles are arranged according to the three areas described in the prior section: measurement frameworks, community engagement in AI development, and AI's role in the protection of community well-being. All these areas have many dimensions to consider and there are also many others that are not included. However, these three provide a foundation for exploring $\mathrm{AI}$ and community well-being in the areas that are most likely to be prevalent or be important in connections efforts and we have selected them as the basis for organizing this special issue.

\section{Measurement Frameworks}

Numerous issues abound as might be expected when devising measurements. Why is this the case? Most simply, it is because what we measure is what we value, such as when funding and other decisions are made on the basis of what is yielded by measurements. At the same time, measurement frameworks may have unintended consequences that are difficult to predict. Communities are dynamic. This leads to the need for dynamic systems enabling new kinds of participatory community-driven measurement frameworks. Similarly, AI system evaluation metrics should not be seen as static artifacts and instead as part of a dynamic and multifaceted process. For the sake of community well-being in the context of AI, data ownership and other issues including but not limited to bias, governance, security, transparency and trust, also need to be addressed as ethical and other considerations can easily arise.

Considering recommender systems presents a starting place for academics and practitioners. This raises questions such as, what is the extent that entities use AI to influence people? Have businesses, nonprofits, educational institutions and governments considered their impacts on community well-being? How can these impacts be measured? The first article, "Aligning AI Optimization to Community Well-being," (Stray 2020) points out that absent incentives to measure and care about community well-being, whether that be public opinion and trust, corporate goodwill, or financial incentives, many entities are unlikely to integrate community well-being into their recommender system objectives. Adding to this hurdle is the lack of research and thus knowledge available about how to accurately and usefully integrate community well-being objects into recommender systems in both the academic and business spheres.

Three articles focus on measurement frameworks (Stray 2020; Ducao et al. 2020; Pettini 2020). Each of the articles that focus on measurement frameworks explored a separate aspect or aspects of well-being within a framework of specific applications. Stray's article, "Aligning AI Optimization to Community Well-being" contemplates community well-being goals and indicators for recommender systems (such as those used by online shopping and news feeds) in terms of objectives. Stray analyzes two case studies, Facebook and YouTube, and provides recommendations for integrating community well-being objectives into recommender systems but notes that absent 
financial incentives or regulations, most entities are not likely to include, much less contemplate, objectives related to community well-being in their recommender systems.

Stray used data gathered from social media posts by Facebook representatives and a book about YouTube. The analysis focuses on the use of metrics for understanding impacts on well-being and that could be used to manage impacts on community wellbeing: meaningful social interactions for Facebook and user satisfaction for YouTube. A recommender system to understand and manage the impact on community wellbeing is important and is guided by many objectives. Community well-being could be incorporated into its objectives through metrics that measure impacts on community well-being (such as climate friendly shopping and diverse perspectives in news feeds).

Ducao, Koen, Gua, Frank, Willard and Kam's article "Multimer: Modeling Neurophysiological Experience in Public Urban Space" is focused on means for gathering biometric data, with some discussion about how to use it for community well-being. Ducao et al. indicate that gathering biometric data from community members via devices they already own, such as smartphones and wearable devices is more efficient than when gathered by potentially more sensitive but difficult to administer machines, such as electrocardiogram (ECG) and other monitors. The maps formulated from the data and a use of them by a nonprofit are presented, as well as discussion of the challenges to privacy, ethics, and other issues in biometric data collection. These biometric data were collected using ECG and other monitors, cell phones, and wearable devices from New York City community members as they traversed various areas in their community on foot or bicycle or in a car. Geographic locations were collected in tandem with the biometric data. The data were fed into a system devised by the author called the Multimer Data Collection and Analysis System (MDCAS) for in-depth analysis presented largely in map form, which could be useful for urban planners, city managers, architects and others who make decisions about the built environment. Ducaso et al.'s MDCAS approach was used by the United Nations Human Settlements Programme (UN-Habitat) to understand walkability, bus routes, and bike lanes and to engage community members in decisions.

Ducao et al.'s collection and analysis of biometric data presents a way to understand the impact on community members of an urban environment and a means to measure that impact through biometric data. However, there are many challenges to collecting and using biometric data for community well-being in design and decision-making processes for urban environments These challenges include data analysis methods as well as presentation and use of the analysis. In addition, Ducao et al. point out that biometric data is deeply personal and could easily be misused. Collection of such data presents many challenges, ranging from trust and data governance to ethics and security. They suggest that community members may be more easily engaged and more willing to contribute to data collection when projects are led by entities that have strong public trust such as the UN-Habitat. In the meantime, most cell phones, and wearable devices are collecting some forms of biometric data, which gives rise to the need for a deeper understanding of the role of community members in the collection of their biometric data and ways for the collection and use of that data to enhance or safeguard community well-being.

Pettini's article "Real-time Assessment of the Burden on the Community of Informal Caregivers: A Pilot Study" explores the feasibility of collecting well-being data from 
informal caregivers via digital devices for studies to understand the impact on wellbeing of caregiving and, potentially for development of AI to improve the well-being of informal caregivers. The data were collected using digital daily diaries (DDD) operationalized through smartphones, as well as through surveys and interviews at the onset and finalization of the pilot study. Pettini's findings suggest that the DDDs were an effective tool. Recommendations include use of the data gathered from DDDs for AIbased strategies and interventions aimed at protecting and improving the well-being for the community of informal caregivers to mentally ill children.

While Pettini's study is limited to the parent caregivers of mentally ill children, it has, in our view, application to a panoply of informal caregivers. Approximately one quarter of the United States (US) population is classified as caregivers (Anderson et al. 2013). We suspect that the percentage of caregivers in many other nations is larger. We also suspect that the percent of the populations that are informal caregivers will grow as the population of the aging community grows (US Census Bureau 2020). In addition, a significant number of parents and family members are contributing caregiving time during the pandemic, with the advent of online schooling. The gathering of data through cell phones and other devices and means such as a DDD and the use of that data to understand and manage the impacts on the well-being of informal caretakers is an area of research that is both nascent and necessary.

\section{Community Engagement in Al Development}

Next, we shift to the issue of community engagement. An invited essay by Havrda, "Artificial Intelligence's Role in Community Engagement within the Democratic Process", presents a method by which AI can foster community engagement in the democratic process and provides steps for ensuring communities are engaged. The two articles about community engagement present concepts that are in use, along with discussing them in the context of AI and community well-being (Hollander et al. 2020; Brown and Ruster 2020). Community engagement is a foundational underpinning of community well-being and related aspects such as community development. It is crucial that the people who live in a place have representation and voice in the decisions made that impact and influence them (Phillips and Pittman 2015). It is essentially a way for residents and other stakeholders to express their opinions, ideas and values. Without people in the equation, it is not community development.

Why is it so important to include foundational concepts such as engagement? First and foremost, the development of AI that has wide-spread impacts will surely influence many dimensions of community life. Considering and including domains of community well-being in the design as well as values of AI will help mitigate negative impacts on people and their communities.

The first of these articles in this section focuses on the vitally important concept of engagement in the development, deployment and management of AI. Hollander, Potts, Hartt and Suti's article, "The Role of Artificial Intelligence in Community Planning" identified the subject of interference in the process of agencies engaging community members in land development and planning processes and decisions. Hollander et al. point out that while there has been some focus on the use of AI to interfere in national elections, the same sort of bad behavior occurs at the community level, and must be acknowledged, brought to light and addressed. 
Their article focuses on the dangers of intentional distortion and influence on processes in which social media is used to engage communities in land or urban development and planning.

They point out the dearth of consideration or exploration into interference with what is intended to be a democratic process of engaging or involving community members in such issues as area planning, policy making, and other issues, listing some of the ways AI can be used to influence and distort the community participation and outcomes of decisions through bots (e.g., astroturfing, twitter bombs, tagging of influential users and spamming). Two recommendations arise: (1) increase capacity to detect and label AIbased bots and (2) encourage officials who are using social media for community engagement to regularly and frequently post clear information about the matter at hand.

Hollander et al.'s assessment and suggestions have implications for other agencies, whether governmental, nonprofit or other sectors, for the use of social media to engage and involve communities in decisions and activities that affect those communities. While there has been extensive research as well as scrutiny of the use of AI and other efforts to interfere in national electoral and other activities, there has been little effort at local levels to understand or mitigate similar activities. With the pandemic, the use of social media will likely be used by many governmental agencies and other entities, bringing to the forefront the urgency of the authors' call for future research on a wider scale. These include studies to understand the scope and impact of AI in community engagement for planning and other purposes and means for managing the impact of AI on processes that involve community members.

Brown and Ruster's article, "Termination for Cultural Misalignment: Setting Up Contract Terms to Ensure Community Well-being in the Development of AI" identifies problems in contracts between Indigenous peoples and governments. In agreements regarding AI, it is important to present clauses for inclusion to protect Indigenous people's rights and ensure equal power between contracting parties. The potential of AI projects precluding considerations around equity, inclusion and justice is immense as profits motives may override social mission. We can already observe this happening in the development of some proprietary algorithms the "corporatization of code" without transparency of what is included; this has and may again lead to negative outcomes such as racial injustices in job applications, and other processes that influence or are used by many in society (K. Dilworth, personal correspondence, September 20, 2020).

The authors use the case of AI technology development projects designed for Indigenous communities among the Aboriginal and Torres Strait Islander peoples of Australia, with the focus of their discussion being the development of a governmentfunded chatbot to encourage smoking cessation in an Indigenous community. They set the context for their discussion by examining the ways in which government agencies and AI technology developers can develop productive collaborative partnerships as they engage with Indigenous community leaders and members, by attending to the cultural worldviews, methodologies, practices and knowledge of the Indigenous communities with which they work. To support this context, they propose the introduction of certain terms and mechanisms into contracts agreed to between Indigenous peoples and government agencies for AI projects. A specific term they identify for protecting Indigenous community well-being is the creation of a termination for cultural misalignment clause within contractual agreements, that recognizes Indigenous rights to 
data sovereignty as laid out in the United Nations Declaration of the Rights of Indigenous Peoples (2007).

Brown and Ruster present two hypothetical scenarios providing comparative examples for the development of contractual agreements meeting proposed requirements. The first example concerns a standard contractual agreement without a termination for cultural misalignment clause, resulting in a process of development that does not engage fully with Indigenous community practices and knowledge to address wellbeing, and ultimately fails. The second example outlines a contractual agreement that includes a termination for cultural misalignment clause that guides and supports a collaborative process of development that draws specifically on the above Declaration (2007) and engages with an Indigenous Cultural Advisory Committee, the members of which represent a range of perspectives, knowledges and practices in the Indigenous communities participating in the program being developed.

As the authors note, establishing this legal mechanism requires recognition and an understanding of Indigenous world views and practices. It also enforces relevant adaptations in the skills, training and cultural competencies of government agencies and AI technology developers engaging with Indigenous communities. This clause provides a framework for developing and establishing guiding principles that protect and ensure Indigenous community well-being. The authors contend that a mechanism that creates collaborative contexts and contractual frameworks that can ameliorate cultural misalignment, and deepen the cultural competency of the contracting parties, leads to more productive collaborations.

\section{Al's Role in the Protection of Community Well-Being}

There are many aspects to consider in protecting community well-being, both from AI and with AI. What do we mean by this? For example, there are power imbalances in the interactions between different communities, academia, industry and government, especially in marginalized communities. Issues of equity and social justice can arise quickly in these contexts and one has only to look as far as the Internet to see many cases where this happens. Lack of access to technology, and lack of due process and participatory avenues to assess and mitigate AI risks abound. Apart from issues around participation (particularly the inability to do so), there is also not enough awareness of the negative externalities and impacts of AI on people and their communities. These and other issues need addressing and further development of AI must take these into consideration.

This is our final article section, concluding with two selections focusing on the role of AI in protecting or improving community well-being (Narayan 2020; Solomon and Baio 2020). These articles delve deeply into theoretical underpinnings of social and economic systems, presenting a wide range of material for thought. While these selections are far from typical or what may be expected in a special issue focused on community well-being, they are included because they provide rich material and multiple complex issues that could serve to inspire future research.

Narayan's essay, "Leveraging Digital Intelligence for Community Well-Being” investigates how well-being is defined within current AI models of economic growth. The author seeks to define a framework that illustrates how AI might be a resource for initiatives focused on improving community well-being. The article examines how technology development that focuses on economic growth connects with community 
well-being, and concludes that assessing well-being using perceptive and experiential measures points to the need to focus on relationality as an important element in defining well-being. The author finds that subjectivity is not limited to individuals, and that subjective actions are not limited to individual responses but emerge from how community groups interact and engage with each other. On this basis, it is suggested that AI initiatives need to move from a focus on individual experience to a focus on how groups of people interact and how those multiple interactions, in terms of contexts and locations, shape people's perceptions and how they relate to the information (and products) they encounter or that are presented to them.

Narayan explores the concept of assemblage that helps to understand diverse aspects of human life and sees potential in the ways that AI, as a tool, could map and situate the many layers of community-based human relatedness. By broadening understandings of the multiple knowledges and practices that exist, and are exchanged or shared, in diverse community contexts, AI could identify those aspects of well-being that community members relate to, value and support. Two models are outlined that offer useful frameworks for leveraging such AI data sources for community well-being. The first model, a knowledge map, illustrates how a diverse range of knowledges might be brought together to reveal their existing connections, in ways that establish how they might relate to insights and perspectives that help to define community-based practices of well-being. The second model offers a framework providing a source of digital intelligence based on data capable of identifying multiple factors that shape and represent community well-being within three specific dimensions: material, relational, and subjective. This framework offers two approaches to a) defining relational perspectives of well-being and the contexts in which they arise, and b) revealing discourses that may both define and limit understandings of the nature of well-being. The overall purpose of engaging with the two models is to provide detailed analyses of the knowledge systems that currently shape understandings about well-being in order to design and develop AI initiatives that can be more closely aligned with community needs.

Perhaps the most creative and provocative article in this special issue, Solomon and Baio's article, "An Argument for an Ecosystemic AI: Articulating Connections across Prehuman and Posthuman Intelligences" suggests biomimicry as a means for the development of AI drawn from art projects involving a type of fungus commonly known as slime mold (Physarum polycephalum). Analysis of the art projects touches on vastly wide-ranging issues to arrive at a proposition for radical change in economic and social systems implemented through AI. Solomon and Baio use the natural growth patterns of the slime mold as a model for the development of AI. Their assumptions include that natural systems, as exemplified by mitochondrial development, are inherently helpful and beneficial to community well-being, and that current economic and social systems based on such goals and metrics as economic growth and gain are harmful to the well-being of communities as well as ecological, social and personal systems. They draw from a trans-disciplinary, holistic and fearlessly revolutionary approach to propose a revolution in AI development.

Solomon and Baio's article draws into question the psychological and societal beliefs, values and assumptions that underpin the current economic, business and social systems in which AI is developed. Their work presents a model for the future development of AI based on biomimicry in which the well-being of human and non- 
human communities is primary. Their discussion also begins to outline a treatise on the dysfunction of business and academic institutions, as well as personal and social systems, from the perspective of revolutionary artists, calling for radical change in the way we think and act and how we value each other, other beings and ecosystems, towards relational, inter and intra-connected and a networked oneness.

\section{Call to Action}

There is abundant experience in the United States and elsewhere indicating that technology, including AI, has tremendous capability to transform positively or negatively our personal lives, governments and business processes (OECD 2018) and consequently our communities. The intent of this special issue is to inspire and promote "a new area of research... to ensure AI reaches its potential to help rather than harm community well-being" (Musikanski et al. 2020a, b, p. 51).

The articles in this special issue represent some preliminary efforts to understand the multi-faceted aspects of what a field of research into the intersections of AI and community well-being could include. They give glimpses into a spectrum of possibilities and show there is a great deal of opportunity to further develop both the definition and dimensions of this field. Our vision is that the field will be cross-sector and interdisciplinary and also integrate a systems-based approach. We recognize the difficulties in this vision and that there are many challenges to crosssectional research, not the least of which is that much AI developed and used by the commercial and governmental sectors is proprietary. Also, there are serious issues involving ethics, trust, transparency, privacy, data governance, data ownership and other factors. However, we believe that through cross-sector research, some of these challenges facing commercial and governmental agencies, will be better understood, which in turn will help protect and benefit community wellbeing. Similarly, we recognize that taking a systems-based approach to this new field of research presents both the unique challenge of confusing systems of AI with systems-based approaches and the larger challenge of taking non-reductive (more complex) perspectives by some researchers accustomed to reductive (more straightforward or simplistic) processes.

However, we must try. Our futures depend on it and certainly community well-being will be highly influenced by further AI development, impacting many domains of our lives, individually and collectively. Perhaps an initial step is encouraging community well-being researchers and practitioners to develop indicators that AI practitioners can use to understand impacts on community well-being and guide the development and deployment of AI. The field of community well-being indicators, with its developed knowledge base of research and applications, could provide a foundation for this step. A review by Musikanski of the well-being chapter in the IEEE publication, "Ethically Aligned Design, and standard 7010-2020 Recommended Practice for Assessing the Impact of Autonomous and Intelligent Systems on Human Well-being", is included in this special issue to provide concepts, methods and indicators that can aid researchers and practitioners in developing indicators. Optimally, researchers will have the opportunity to work in partnership with the companies and agencies creating and using AI, but regardless we hope that there will be research into the multi-dimensional impacts of 
AI on community well-being and how they can be measured. This is important because measurements, such as indicators, can help foster understanding, management and guiding of the influence of AI on community well-being, and hopefully, set goals to positively impact and protect communities.

We deeply believe in community-based approaches to any solution, whether it be a project, program or policy impacting community well-being. There is a great deal of obscurity in the development of AI, compounded by the lack of clarity by many people about what it is and is not. There is a great need to involve communities in the process of development and deployment of AI, from its ideation phase to its implementation, monitoring and revision or replacement. We understand how complex this call to action is and suggest following rethinking contracts, ranging from common everyday agreements on the use of an AI product to more interactive arrangements in the development phases of AI to include clauses that preserve and protect community well-being along its many dimensions (Brown and Ruster, 2020). In addition, we hope that companies and governmental agencies will work with the communities affected by the AI they create or deploy in the development of products, as well as management and maintenance of products, projects or policies in which AI plays any part.

Last but by no means least, we call on all companies and other entities using or developing AI to allocate resources towards the development of AI to help solve the seemingly overwhelming challenges facing humans and our community well-being now and for future generations. These challenges include those current in our lives today, such as better management of the pandemic; the effects of climate change with wildfires, hurricanes and flooding on people and other species; interference in elections and the processes of democracy; and spreading infection of hatred for one's neighbor and fellow resident in our communities. We believe that AI is part of the solution for a world we all want to live in, for communities we all want our children and grandchildren to enjoy - communities where all people flourish, indigenous wildlife is abundant, natural resources are plentiful, economies are dependable and equitable, and societies are happy and flourishing.

\section{References}

Anderson, L. A., Edwards, V. J., Pearson, W. S., Talley, R. C., McGuire, L. C., \& Andresen, E. M. (2013). Adult caregivers in the United States: Characteristics and differences in well-being, by caregiver age and caregiving status. Preventing Chronic Disease, 10, E135. https://doi.org/10.5888/pcd10.130090.

Maani, K., \& Cavana, R. (2007). Systems thinking, system dynamics: Managing change and complexity. North Shore: Pearson Education.

McGregor, J. (2007). Research well-being: From concepts to methodology. In I. Gough \& J. A. McGregor (Eds.), Well-being in developing countries: From theory to research (pp. 316-355). New York: Cambridge University Press.

Musikanski, L., Phillips, R., Bradbury, J., De Graaf, J., \& Bliss, C. (2020a). Happiness, well-being and sustainability, a course in systems change. London: Earthscan/Routledge.

Musikanski, L., Rakova, B., Bradbury, J., Phillips, R., \& Manson, M. (2020b). Artificial intelligence and community well-being: A proposal for an emerging area of research. International Journal of Community Well-Being, 3(1), 39-55. https://doi.org/10.1007/s42413-019-00054-6.

Organization for Economic Cooperation and Development (OECD) (2018). Going digital in a multilateral word. Paris: OECD Publishing. Retrieved September 30, 2020 from https:/www.oecd.org/going-digital/ C-MIN-2018-6-EN.pdf. Accessed 1 Sept 2020. 
Organization for Economic Cooperation and Development (OECD) (2019). OECD better life initiative: measuring well-being and progress. Retrieved October 13, 2020. https:/www.oecd.org/statistics/betterlife-initiative.htm [Online Resource]. Accessed 5 Sept 2020.

Phillips, R., \& Pittman, R. (2015). Introduction to community development (2nd ed.). London: Routledge. Phillips, R., \& Wong, C. (2017). Handbook of community well-being research. Dordrecht: Springer.

U.S. Bureau of the Census (2020). 65 and older population grows rapidly as baby boomers age. Retrieved September 30, 2020 from https:/www.census.gov/newsroom/press-releases/2020/65-older-populationgrows.html [Online resource]. Accessed 3 Sept 2020

Publisher's Note Springer Nature remains neutral with regard to jurisdictional claims in published maps and institutional affiliations.

\section{Affiliations}

\section{R. Phillips ${ }^{1} \cdot$ L. Musikanski ${ }^{2} \cdot$ M. Manson ${ }^{3} \cdot$ J. $_{\text {Bradbury }}{ }^{2} \cdot$ L. Fraizer $^{4} \cdot$ B. Rakova ${ }^{5}$. N. DePalma ${ }^{6} \cdot$ A. Smart ${ }^{7}$}

L. Musikanski

laura@happycounts.org

M. Manson

mmanson174@gmail.com

J. Bradbury

jnbrad71@gmail.com

L. Fraizer

1fraizer@alumni.cmu.edu

B. Rakova

b.rakova@gmail.com

N. DePalma

ndepalma@gmail.com

A. Smart

andrewsmart@google.com

Purdue Honors College, West Lafayette, IN, USA

2 Happiness Alliance, Seattle, WA, USA

3 Independent Researcher, IN, USA

4 University of the Pacific, Stockton, CA, USA

5 Accenture Responsible AI, San Francisco, CA, USA

6 Facebook AI, Pittsburgh, PA, USA

7 Google, San Francisco, CA, USA 